\title{
Ultrafast conversions between hydrogen bonded structures in liquid water observed by femtosecond $x$-ray spectroscopy
}

\author{
Haidan Wen, ${ }^{1 \neq}$ Nils Huse,${ }^{27^{*}}$ Robert W. Schoenlein ${ }^{2,3}$ and Aaron M. Lindenberg ${ }^{1,4^{*}}$ \\ ${ }^{1}$ PULSE Institute, SLAC National Accelerator Laboratory, Menlo Park, CA 94025, USA \\ ${ }^{2}$ Ultrafast X-ray Science Laboratory, Chemical Sciences Division, Lawrence Berkeley National Laboratory, \\ Berkeley, CA 94720, USA \\ ${ }^{3}$ Material Sciences Division, Lawrence Berkeley National Laboratory, Berkeley, CA 94720, USA \\ ${ }^{4}$ Department of Materials Science and Engineering, Stanford University, Stanford, CA 94305, USA
}

DOI: $10.1063 / 1.3273204$

$\$$ These authors have contributed equally to this work.

*Authors to whom correspondence should be addressed: aaronl@stanford.edu and nhuse@lbl.gov

\begin{abstract}
We present the first femtosecond soft x-ray spectroscopy in liquids, enabling the observation of changes in hydrogen bond structures in water via core-hole excitation. The oxygen K-edge of vibrationally excited water is probed with femtosecond soft $\mathrm{x}$-ray pulses, exploiting the relation between different water structures and distinct $\mathrm{x}$-ray spectral features. After excitation of the intramolecular $\mathrm{OH}$ stretching vibration, characteristic x-ray absorption changes monitor the conversion of strongly hydrogen-bonded water structures to more disordered structures with weaker hydrogen-bonding described by a single sub-picosecond time-constant. The latter describes the thermalization time of vibrational excitations and defines the characteristic maximum rate with which non-equilibrium populations of more strongly hydrogen-bonded water structures convert to less-bonded ones. On short time-scales, the relaxation of vibrational excitations leads to a transient high-pressure state and a transient absorption spectrum different from that of statically heated water.
\end{abstract}

\section{INTRODUCTION}

The ubiquity of water in nature has stirred great research interest in many fields of science. In particular, molecular processes in living organisms exploit and depend on the unusual properties of water. Liquid water can be regarded as a highly dynamic network of molecules connected through hydrogen bonds. ${ }^{1}$ The dynamics of these bonds is largely responsible for the unique properties of water and poses significant challenges for theoretical modeling and experimental investigations. In a new approach, we apply the emerging technique of femtosecond soft $\mathrm{x}$-ray spectroscopy to liquid water.

Previously, a wide range of optical techniques has been employed to investigate the static structural properties of water including vibrational spectroscopy. ${ }^{2-4}$ In recent decades, advances in laser technology have enabled femtosecond vibrational spectroscopy studies of dynamics in water ${ }^{5-9}$ which show that the hydrogen bond network evolves over a broad range of time scales. This gives rise to infrared absorption bands with very complex behavior occurring on time scales well below 100 femtoseconds. ${ }^{10-14}$ The observed dynamics on ultrafast time scales have been successfully identified with: the fast modulation of transition frequencies by coupled molecular oscillators, resonant energy transfer among the densely packed $\mathrm{OH}$ stretching oscillators, and exceptionally efficient vibrational energy relaxation. All of these findings are a manifestation of the fluctuating hydrogen bond network that forms the 'supramolecular' structure of water.

Using much higher photon energies, the absorption spectrum of the oxygen K-edge has been measured with $\mathrm{x}$-ray Raman scattering (XRS) and x-ray absorption spectroscopy
(XAS) via transmission, fluorescence and total/Auger electron yield (TEY/AEY). ${ }^{15,16}$ The results of these static measurements have been combined with theoretical treatments in an attempt to produce quantitative structural models of liquid water. $^{17,18}$ This approach has tried to link the pre-edge (I), main-edge (II) and post-edge (III) regions indicated in Fig. $2 \mathrm{~A}$ to local water structures with specific coordination and hydrogen bonding. The results of the different theoretical approaches, and more detailed interpretations of experimental data, have led to significant controversy over the correct structural model and their relative merits in determining the average number of hydrogen bonds per water molecule. Nevertheless, there is consensus on a qualitative level as to the structural meaning of the three characteristic spectral regions of the oxygen K-edge, and how these features change upon melting of ice as well as heating of liquid water. Specifically, an absorption decrease in the post-edge region is associated with a decrease of highly coordinated water structures while the pre- and main-edge absorption is indicative of weakly hydrogen-bonded water structures. ${ }^{17-22}$ More detailed interpretations of experimental data based on specific water models have led to much discussion about the relative merits of various models. Very recently, transient XAS has been demonstrated ${ }^{23,24}$ but the sub-nanosecond time resolution of these experiments precluded observations at relevant time scales. Further research is needed to develop an understanding of hydrogen bonding in water that is consistent with the broad range of experimental observations.

Femtosecond x-ray spectroscopy is a powerful new tool for investigating structure and dynamics simultaneously. It provides new information about nuclear motions on their fundamental time scales, combined with the high chemical 
Wen et al.

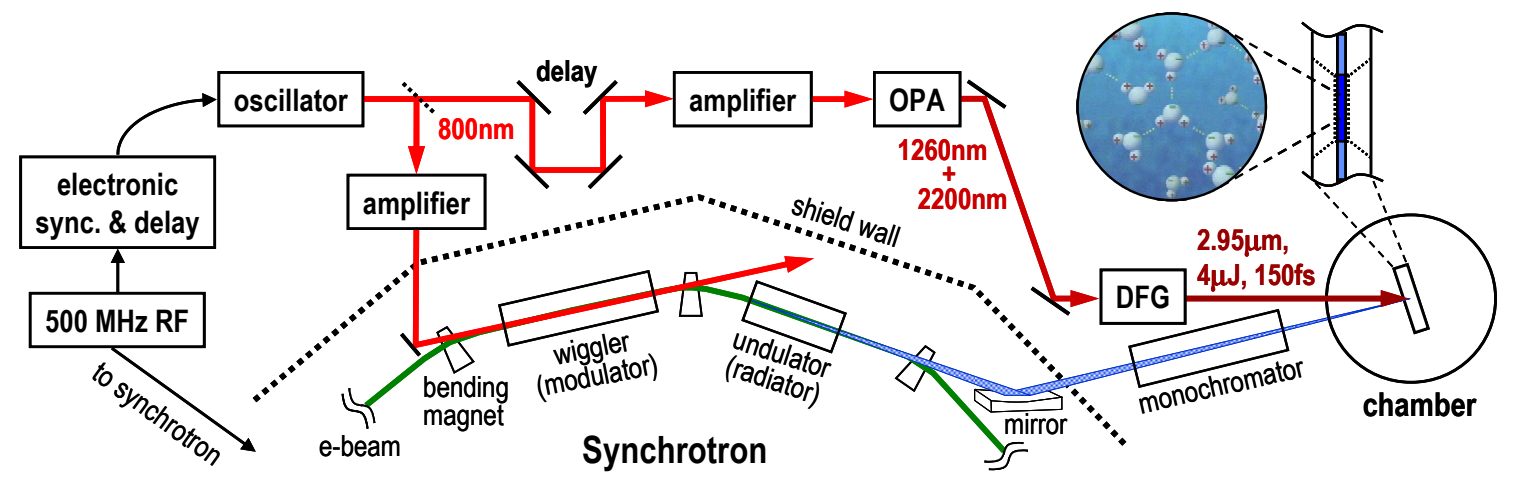

FIG. 1. Schematic drawing of the femtosecond soft x-ray beamline at the Advanced Light Source. A femtosecond Ti:Sapphire laser system supplies two synchronous laser pulses. One of them is sent into the synchrotron to generate a femtosecond $\mathrm{x}$-rays pulse, the second pulse is frequency-converted to the infrared via an optical parametric amplifier (OPA) with subsequent difference frequency generation (DFG) and is used for excitation of the $\mathrm{OH}$ stretching mode in an infrared pumplx-ray probe experiment.

specificity and the sensitivity to local structure on atomic length scales. In this work, we present femtosecond x-ray spectroscopy of vibrationally excited water which probes the ultrafast response of core-hole transitions to changes in hydrogen bonding, to our knowledge the first femtosecond experiment in any solution phase system in the soft $x$-ray range.

\section{EXPERIMENTAL}

We use a novel femtosecond soft x-ray beamline at the Advanced Light Source as depicted in Fig. 1. To investigate the structural rearrangements in liquid water, we resonantly excite the $\mathrm{OH}$ stretching vibration of the water molecules with femtosecond infrared pulses tuned to $2.95 \mu \mathrm{m}$ wavelength, and directly probe the subsequent dynamics of the hydrogen bond network by femtosecond $\mathrm{x}$-ray absorption near-edge spectroscopy (fs-XANES) at the oxygen K-edge shown in Fig. 2A. The static x-ray absorption spectrum is obtained by integrating the $\mathrm{x}$-ray induced current in a Si photodiode while scanning the $\mathrm{x}$-ray energy across the oxygen $\mathrm{K}$ edge with a $0.5 \mathrm{eV}$ resolution.

The picosecond differential $x$-ray absorption spectrum in Fig. $2 \mathrm{~B}$ is measured with a flux of about $10^{3}$ photons/pulse $/ 0.1 \%$ bandwidth and a data acquisition rate of 1 $\mathrm{kHz}$ at a time delay of $150 \mathrm{ps}$ between the infrared pump pulses and the $70 \mathrm{ps} x$-ray pulses directly provided by the synchrotron. The infrared pulses are generated via difference frequency generation of the output of an optical parametric amplifier (TOPAS-C, Light Conversion) that is pumped at $1.6 \mathrm{~mJ}$ with $70 \mathrm{fs}$ pulses at $800 \mathrm{~nm}$ wavelength. The $800 \mathrm{~nm}$ pump pulses are provided by a $1 \mathrm{kHz}-\mathrm{Ti}$ :Sapphire laser system that is seeded by a cavity-length-stabilized oscillator at $62.5 \mathrm{MHz}$ (synchronized to the $500 \mathrm{MHz}$ radio frequency of the synchrotron's storage ring). The infrared pump pulses with $4 \mu \mathrm{J}$ energy are focused to $150 \mu \mathrm{m}$ in diameter and overlapped with the $50 \mu \mathrm{m} x$-ray probe pulses on the sample at a crossing angle of $\sim 10^{\circ}$. The $\mathrm{x}$-ray pulses are modulated by a chopper at $2 \mathrm{kHz}$ to which the laser amplifiers are synchronized. The delay between infrared pump and $\mathrm{x}$-ray probe pulses is varied electronically. The $\mathrm{x}$-ray pulses transmitted through the sample are detected with a suitable gate, intermit- tently recording transmitted $\mathrm{x}$-ray intensities with and without the infrared pump pulses, $\mathrm{I}(\mathrm{t})$ and $\mathrm{I}_{0}$, to calculate absorbance changes as a function of pump-probe delay $\mathrm{t}, \Delta \mathrm{A}(\mathrm{t})=$ $\log \left(\mathrm{I}(\mathrm{t}) / \mathrm{I}_{\mathrm{o}}\right)$.

In order to improve the time resolution of the transient $\mathrm{XAS}$, femtosecond $\mathrm{x}$-ray pulses are derived from the interaction between a dedicated femtosecond laser pulse and one of the electron bunches in the synchrotron storage ring. ${ }^{25} \mathrm{~A} 70$ fs pulse at $2 \mathrm{~mJ}$ energy and $800 \mathrm{~nm}$ wavelength is sent into the synchrotron at $1 \mathrm{kHz}$ and co-propagates with the electron beam through the wiggler (modulator) to create a slice of femtosecond electrons which subsequently radiates (in the undulator) a femtosecond $\mathrm{x}$-ray pulse that is spatially displaced from the picosecond pulse and isolated by a pair of slits in the image-plane of the beamline. We record an average femtosecond x-ray flux at the sample position corresponding to several photons per laser pulse in $0.1 \%$ bandwidth. The residual intensity of the picosecond pulses serves as a reference to normalize the absorption change detected with femtosecond pulses. The infrared pump pulses and the $\mathrm{x}$-ray probe pulses are intrinsically synchronized because they originate from the same seed pulse. The time delay between pump and probe pulses is controlled by a steppermotor stage that changes the optical path length of the pump pulses. The time-resolution is determined by the $200 \mathrm{fs} x$-ray pulses as the infrared pump pulses are $\sim 100 \mathrm{fs}$.

Due to the large absorption cross-sections of the $\mathrm{OH}$ stretching vibration and soft x-ray transitions in general, stable ultra-thin liquid films in a vacuum-type environment under typical laser excitation conditions are a significant experimental challenge. An ultrathin liquid cell with controllable thickness is used to create a thin liquid water film with adjustable film thickness. ${ }^{14,24}$ The liquid film is sandwiched between two flexible $100 \mathrm{~nm}$-thick silicon nitride (SiN) membranes separated by two $1.8 \mu \mathrm{m}$-thick spacer pads of photoresist on one of the substrates that hold the SiN membranes. The spacer pads create a $500 \mu \mathrm{m}$ wide channel into which the fluid is drawn by capillary forces. The SiNassembly is held in a sample cell that is connected via tubing to the exterior of the experimental chamber in which it is mounted to allow for control of the cell's interior pressure. 


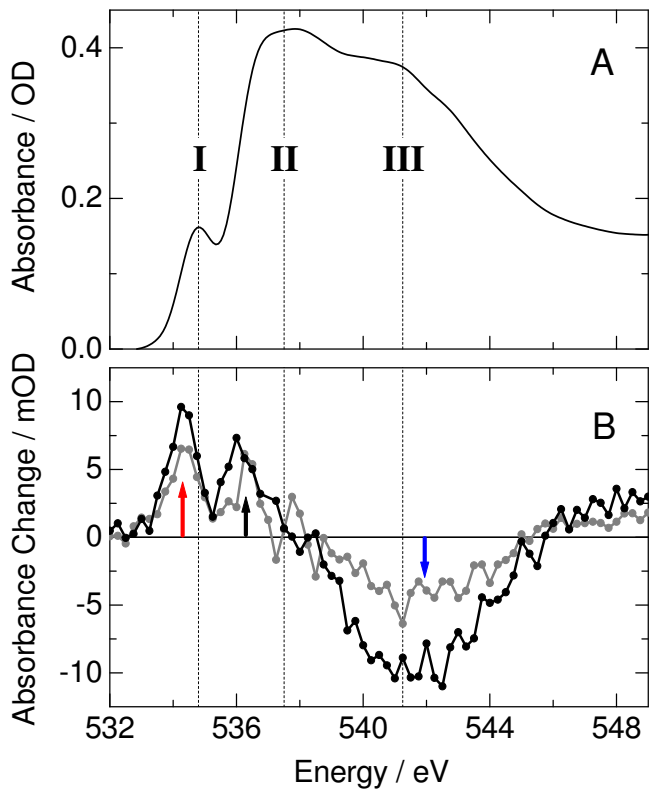

FIG. 2. Static and differential absorption spectra. A Static oxygen x-ray absorption spectrum of a $400 \mathrm{~nm}$ thick water film. Dashed lines mark local absorption maxima which define the characteristic spectral regions: the preedge (I), main-edge (II), and post-edge (III) of the water x-ray spectrum. B Differential absorption spectra of the oxygen XANES region recorded at 150 ps (black) and $300 \mathrm{~ns}$ (gray) delay. Arrows indicate the spectral positions at which pump-probe transients are recorded (cf. figure 3).

The experimental chamber itself is filled with helium at 150 Torr to offset the vapor pressure of the liquid inside the sample cell. The flexibility of the silicon nitride membranes permits sample thicknesses from below $200 \mathrm{~nm}$ to tens of microns by tuning the relative pressure between the cell interior and the experimental chamber.

\section{RESULTS}

First, we present absorbance changes of the XANES region as a function of energy at a delay of $150 \mathrm{ps}$ in Fig. 2B. This data is recorded with picosecond $\mathrm{X}$-ray pulses provided directly by the synchrotron. The differential spectrum shows a pronounced absorption increase at the low-energy sides of the pre-edge $(534.3 \mathrm{eV})$ and the main edge $(536.3 \mathrm{eV})$, while the spectrum features a broad absorption decrease across the post-edge region $(542 \mathrm{eV})$. Beyond $546 \mathrm{eV}$, a transient absorption increase is observed extending to higher energies ( $>556 \mathrm{eV}$, not all data shown). Absorbance changes in the pre- and post edge region are comparable in magnitude, while the maximal changes in the main-edge region are about half as large.

To follow the very fast dynamics in water, we employ femtosecond x-ray pulses and measure the transient absorbance change at three characteristic spectral positions across the oxygen XANES region of water as shown in Fig. 3. These spectral positions lie in the pre-, main-, and post-edge region (cf. Fig. 2) that are sensitive to changes in hydrogen bond configurations. ${ }^{17,18,20}$ While the transient absorption at the pre- and the main-edge increases during the first 3 ps following vibrational excitation, the post-edge absorption decreases simultaneously. The absorption changes at the pre- and post- edges are equal in magnitude but opposite in sign while the maximum absorption change at the main edge is half as large, consistent with the differential spectrum in Fig. 2B. All three transients show similar temporal behavior although the signal-to-noise ratio is enhanced at the pre-edge because of the lower absorption, allowing for better photon statistics for a given absorption change. Over this delay range, the observed dynamics are independent of pump pulse fluence between 20 and $40 \mathrm{~mJ} / \mathrm{cm}^{2}$ and independent of sample thickness, indicating that the observed time scales are intrinsic to liquid water.

To quantify the absorption change in Fig. 3, we model the temporal behavior of the experimental data with simple rate equations and a single time constant for all three spectral positions. The result is included in Fig. 3 as solid lines, illustrating that a time constant of $0.7( \pm 0.2)$ ps describes the absorbance changes across the XANES region very well. For time delays up to hundreds of picoseconds, the evolution of the transient absorption spectrum can be described by the product of a time-independent amplitude function and an exponential time constant such that $\Delta \mathrm{A}(\mathrm{E}, \mathrm{t})=\left[\Delta \mathrm{A}_{\mathrm{o}}(\mathrm{E}) \cdot(1-\right.$ $\left.\left.e^{t / \tau}\right)\right] \otimes R(E, t)$ where $R$ is the experimental response function related to energy $\mathrm{E}$ and time t. $\Delta \mathrm{A}_{\mathrm{o}}$ is then given by the data in Fig. 2B while the sub-picosecond dynamics are described by the time constant $\tau=0.7$ ps.

We do not observe appreciable absorption changes between 3 ps and 1 ns, but on nanosecond time scales further dynamics manifest as is evident from Fig. $2 \mathrm{~B}$ and the insets of Fig. 3. Clearly, the absorption changes in the pre- and postedge region partially recover to magnitudes comparable to that at the main-edge while the latter remains constant. We have modeled this nanosecond behavior by an instantaneous absorption change that recovers exponentially, thereby extracting a time constant $\tau_{\mathrm{E}}=8( \pm 3)$ ns for a sample of $400 \mathrm{~nm}$ thickness. We find that $\tau_{\mathrm{E}}$ increases monotonically when changing the sample thickness from $200 \mathrm{~nm}$ and $800 \mathrm{~nm}^{24}$

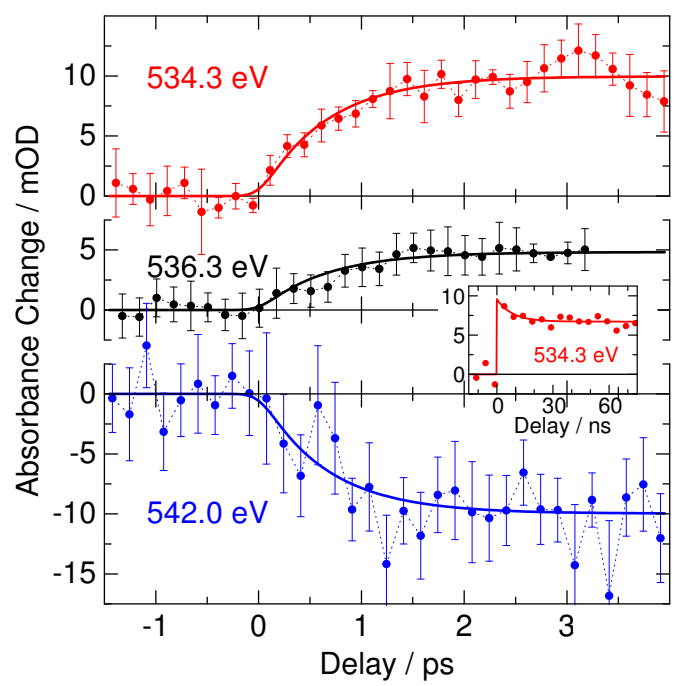

FIG. 3. Absorbance change as a function of time delay between the infrared pump pulses and the x-ray probe pulses at three characteristic spectral positions: $534.3 \mathrm{eV}$ (red), $536.3 \mathrm{eV}$ (black), and $542.0 \mathrm{eV}$ (blue). Solid lines are single exponential fits to the data with a time constant of $0.7 \mathrm{ps}$. The inset shows a time transients on nanosecond time scales recorded on a $400 \mathrm{~nm}$ thick sample, in which the solid red line represents a fit to the data of the form $\Delta \mathrm{A}_{\mathrm{ns}}(\mathrm{E}, \mathrm{t})=\mathrm{C}(\mathrm{E})+\mathrm{D}(\mathrm{E}) \cdot \mathrm{e}^{\mathrm{t} / \tau_{\mathrm{E}}}$ with $\mathrm{C} / \mathrm{D} \approx 2$ and $\tau_{\mathrm{E}}=8( \pm 3)$ ns. $\tau_{\mathrm{E}}$ increases with increasing sample thickness. 


\section{DISCUSSION}

Before discussing the interpretation of the dynamics presented in Fig. 3, we first consider the implications of the spectral changes in Fig. 2. Our work determines changes in the distribution of water structures by probing spectral indicators of molecular coordination at the oxygen K-edge. Altered coordination refers to changes in the average hydrogen bond strength between water molecules. Although the spectral indicators cannot quantitatively measure the hydrogen bond strength and structural changes without the aid of mathematical modeling, they provide qualitative information on water structures such as the deviation from ice-like arrangements. As mentioned before, the absorption in the post-edge region has been associated with ordered and highly coordinated water structures while the pre- and main-edge absorption is considered indicative of weakly hydrogen-bonded water structures. Thus, the loss of absorption in the post-edge region at 150 ps signals a reduction in highly coordinated water structures, while the absorption increase at the pre- and main-edge reflects a decrease in average hydrogen-bond strength. This spectral behavior is indicative of heating as comparison of the differential spectra in Fig. 2 with XRS spectra of water at different temperatures ${ }^{26}$ and transient XAS data shows. ${ }^{23,24}$

The femtosecond transients in Fig. 3 follow the true evolution of these absorption changes, thereby revealing an increase of weakly hydrogen-bonded water structures at the expense of highly coordinated ones. The underlying relaxation of vibrational energy (initially placed in excited $\mathrm{OH}$ stretching vibrations) proceeds with a characteristic time constant of $0.7 \mathrm{ps}$. The latter can be identified with the subpicosecond thermalization time of the hydrogen bond network that has been extracted from femtosecond infrared spectroscopy measurements. ${ }^{27-29}$ Importantly, the dynamics of the hydrogen bond network are directly reflected in the absorption changes of the oxygen K-edge which seems relatively insensitive to excitations of the internal vibrational degrees of freedom (i.e. the stretching and the bending modes of individual water molecules). This is apparent in the subpicosecond dynamics of Fig. 3 when compared to femtosecond vibrational spectroscopy measurements where the sensitivity of vibrational probes to intramolecular modes leads to additional transient signals at delays below $500 \mathrm{fs}$ that reflect the cascade of intramolecular vibrational energy relaxation: from the initially excited $\mathrm{OH}$ stretching mode to the $\mathrm{OH}$ bending mode $(\sim 0.2 \mathrm{ps})$, relaxation of the $\mathrm{OH}$ bend $(\sim 0.2 \mathrm{ps})$, and subsequent thermalization of energy in the hydrogen bond network. ${ }^{28,30,31}$

In water, the thermalization time for vibrational excitations has been interpreted as the average time necessary to change the local hydrogen bonding. Molecular dynamics (MD) simulations of water have defined hydrogen bond lifetimes in terms of a time correlation function that describes the relationship between an initially tagged hydrogen bond and its manifestation at later times. For water in thermodynamic equilibrium, such simulations report hydrogen bond lifetimes of $\geq 1 \mathrm{ps}^{32,33}$ but non-equilibrium conditions such as the vibrational excitations in this experiment should give rise to faster dynamics than predicted by MD-simulations of thermally equilibrated ensembles. This explains why the observed time constant for energy redistribution in the hydrogen bond network is consistently below $1 \mathrm{ps}$, illustrating that vibrational excitations in water decay very rapidly to a thermalized state in which the initially absorbed energy in a single molecule is distributed over a considerable number of molecules within a few picoseconds. We note that this delocalization is not the one discussed in the context of resonant energy transfer. ${ }^{12,14}$ It merely owes to the fact that (i) the initial energy quanta absorbed by $\sim 3 \%$ of the $\mathrm{OH}$ stretching oscillators thermalize to increase the population in the energetically lowest degrees of freedom which are delocalized over several water molecules ${ }^{28,34}$ and (ii) the experiment's initial excitation density translates to an average of $\sim 7$ unexcited water molecules or $\sim 2 \mathrm{~nm}$ between two excited water molecules. Thus the diffusion of thermal energy over length scales as short as $1 \mathrm{~nm}$ are sufficient to achieve thermal homogeneity.

In summary, the transient absorbance changes in Fig. 3 monitor the rearrangement of the hydrogen bond network in water upon relaxation of ultrafast vibrational excitations. In this process, highly coordinated water structures are converted to configurations characterized by weaker hydrogen bonding and less order, as evidenced by the spectral changes of the oxygen K-edge. There is no evidence of an intermediate state (as might be indicated by differing dynamics at the characteristic oxygen K-edge features) thus implying that water structures of lower coordination are created at the direct expense of the highly coordinated structures. A recent xray study using static methods reports micro-heterogeneity due to two types of competing water structures ${ }^{35}$ similar to ideas first put forward by Röntgen and others ${ }^{36}$ and in line with Raman spectroscopic studies. ${ }^{37}$ In such a model, the transients in Fig. 3 would follow the equilibration of the relative abundances of the competing structural species created by an impulsive energy increase with an associated conversion rate constant of $1.4 \mathrm{ps}^{-1}$.

The rapid sub-picosecond dynamics shown in Fig. 3 lead to isochoric heating of the water sample, resulting in a simultaneous increase of temperature and internal pressure. This interpretation follows directly from the sample expansion time for which a lower time limit of $\sim 300$ ps is given by the sample thickness of $400 \mathrm{~nm}$ and the hydrodynamic speed of sound. Thus, essentially no expansion occurs within the measured $0.7 \mathrm{ps}$ thermalization time. The heated water volume expands adiabatically on nanosecond time scales, similar to the behavior of ice. ${ }^{24,38}$ This is evidenced by the partial absorption recovery at nanosecond delays, seen in Fig. 2 and the inset of Fig. 3. Therefore, the differential absorption spectrum on sub-nanosecond delays bears information on changes in hydrogen bonding due to both pressure and temperature. The magnitude and sign of the femtosecond changes in the $\mathrm{x}$ ray absorption spectrum at the three measured energies are in good agreement with the full transient absorption spectrum measured at $100 \mathrm{ps}$ and thus indicate that the $100 \mathrm{ps}$ transient absorption spectrum is a close approximation of the transient spectrum at all delay times $t>0$ fs. From the experimental parameters, we estimate a rise in temperature of $20 \mathrm{~K}$ accom- 
panied by a transient high-pressure phase of $\sim 15 \mathrm{MPa}$. In water above $4^{\circ} \mathrm{C}$, an adiabatic increase in temperature increases the average oxygen-oxygen distance. However, isochorically heated water maintains its initial average density at early times ( $\ll 1 \mathrm{~ns}$ ), as confirmed by time-resolved diffraction studies. ${ }^{39}$ Consequently, changes in hydrogen bonding associated with weaker hydrogen bond interactions must stem from bond distortions (larger bond angles) and increased disorder on these time scales. The observed insensitivity of the main-edge absorption peak to pressure changes at short delay times (high pressures for $\mathrm{t} \ll 1 \mathrm{~ns}$ ) is in good agreement with the observed changes comparing high-density amorphous ice created at 18 bar to ambient water. ${ }^{22}$

\section{CONCLUSIONS}

In conclusion, we present the first femtosecond soft x-ray spectroscopy of liquid water, thereby recording the flow of energy from intramolecular vibrations towards thermal equilibrium with increased intermolecular disorder, manifesting in fewer/weaker hydrogen bonds and structural rearrangements. Upon vibrational excitation, the conversion of highly coordinated water structures to less-ordered structures with weaker hydrogen-bonding is recorded via femtosecond XANES spectroscopy-exploiting the relation between coordination/order and distinct X-ray spectral features due to the high sensitivity of XANES regions to chemical coordination. The observed dynamics are described by a 0.7 ps thermalization time that is characteristic of the hydrogen bond network in water. The transient measurements capture the formation of a high pressure phase distinct from the equilibrium state of liquid water.

Our work is the first femtosecond soft x-ray study of a liquid-phase system. It demonstrates the feasibility and prospects of solution-phase ultrafast $\mathrm{X}$-ray studies in the soft $\mathrm{X}$-ray range where transitions (K-edges of $\mathrm{C}, \mathrm{N}, \mathrm{O}$ and L-edges of transition metals) deliver chemically specific information about coordination, valence charges distribution, bonding, and atomic structure in order to uniquely follow chemical reactions and solvation dynamics on ultrafast time scales.

\section{ACKNOWLEDGEMENTS}

This work was supported by the Director, Office of Science, Office of Basic Energy Sciences, the Chemical Sciences, Geosciences, and Biosciences Division under the Department of Energy Contract No. DE-AC02-05CH11231 (LBNL - Ultrafast X-ray Science Laboratory), by the Department of Energy through the Stanford PULSE Institute, and by the National Science Foundation (NSF) Contract No. CHE-0518637.

\section{LEGAL DISCLAIMER}

This document was prepared as an account of work sponsored by the United States Government. While this document is believed to contain correct information, neither the United States Government nor any agency thereof, nor
The Regents of the University of California, nor any of their employees, makes any warranty, express or implied, or assumes any legal responsibility for the accuracy, completeness, or usefulness of any information, apparatus, product, or process disclosed, or represents that its use would not infringe privately owned rights. Reference herein to any specific commercial product, process, or service by its trade name, trademark, manufacturer, or otherwise, does not necessarily constitute or imply its endorsement, recommendation, or favoring by the United States Government or any agency thereof, or The Regents of the University of California. The views and opinions of authors expressed herein do not necessarily state or reflect those of the United States Government or any agency thereof or The Regents of the University of California.

\section{REFERENCES}

1. D. Eisenberg and W. Kauzmann, The Structure and Properties of Water, Oxford University Press, U. K., 1969.

2. A. K. ÅngSTRÖM, Phys. Rev. 3, 47 (1914).

3. H. R. ZelsmanN, J. Mol. Struct. 350, 95 (1995).

4. Y. Tominaga, A. Fujiwara, and Y. Amo, Fluid Phase Equilib. 144, 323 (1997).

5. H. Graener, G. Seifert, and A. Laubereau, Phys. Rev. Lett. 66, 2092 (1991).

6. S. WOUTERSEN, U. EMMERICHS, and H. J. BAKKER, Science 278, 658 (1997).

7. G. M. Gale, F. H. G. Gallot, N. Lascoux, S. Bratos, and J.-C. LEICKNAM, Phys. Rev. Lett. 82, 1068 (1999).

8. J. C. DeÁk, S. T. RheA, L. K. Iwaki, and D. D. Dlott, J. Phys. Chem. A 104, 4866 (2000).

9. S. Yeremenko, M. S. PshenichniKov, and D. A. Wiersma, Chem. Phys. Lett. 369, 107 (2003).

10. C. J. FeCKo, J. D. EAves, J. J. Loparo, A. TOKMAKofF, and P. L. GEISSLER, Science 301, 1698 (2003).

11. J. B. Asbury, T. Steinel, C. Stromberg, S. A. C. C. P. Lawrence, J. L. Skinner, and M. D. FAYER, J. Phys. Chem. A 108, 1107 (2004).

12. M. L. Cowan, B. D. Brunner, N. HuSE, J. Dwyer, B. Chugh, E. T. J. Nibbering, T. ElsAesSER, and R. J. D. MilLER, Nature 434, 199 (2005).

13. N. Huse, S. Ashihara, E. T. J. NibBering, and T. Elsaesser, Chem. Phys. Lett. 404, 389 (2005).

14. D. Kraemer, M. L. Cowan, A. PaAmmann, N. Huse, E. T. J. NibBering, T. Elsaesser, and R. J. D. Miller, Proc. Natl. Acad. Sci. 105, 437 (2008).

15. B. X. YAng and J. KIRZ, Phys. Rev. B 36, 1361 (1987).

16. L. A. NÄSLUND, J. LÜNING, Y. UfUKTEPE, H. OGASAWARA, P. Wernet, U. Bergmann, L. G. M. Pettersson, and A. NilsSon, $J$. Phys. Chem. B 109, 13835 (2005).

17. P. Wernet, D. NordLund, U. Bergmann, M. CAVAlleri, M. Odelius, H. Ogasawara, L. A. NÄSLUnd, T. K. HiRSCH, L. OJamä̈, P. Glatzel, L. G. M. Pettersson, and A. Nilsson, Science 304, 995 (2004).

18. J. D. SMith, C. D. CAPPA, K. R. Wilson, B. M. Messer, R. C. Cohen, and R. J. SAYKALLY, Science 306, 851 (2004).

19. S. Myneni, Y. Luo, L. Å. NÄSLund, M. CAVAlleri, L. OJAMÄE, H. Ogasawara, A. Pelmenschikov, P. WeRnet, P. VÄterlein, C. Heske, Z. Hussain, L. G. M. Pettersson, and A. Nilsson, J. Phys.: Condens. Matter 14, L213 (2002).

20. B. Hetenyi, F. D. Angelis, P. Giannozzi, and R. CAR, J. Chem. Phys. 120, 8632 (2004).

21. D. Prendergast and G. Galli, Phys. Rev. Lett. 96, 215502 (2006). 
22. J. S. Tse, D. M. Shaw, D. D. Klug, S. PatchkovskiI, G. Vanko, G. MONACO, and M. KRISCH, Phys. Rev. Lett. 100, 095502 (2008).

23. P. Wernet, G. Gavrila, K. Godehusen, C. Weniger, E. Nibbering, T. ElsAesser, and W. EBerhardt, Appl. Phys. A: Mater. Sci. Process. 92, 511 (2008).

24. N. Huse, H. Wen, D. Nordlund, E. Szilagyi, D. Daranciang, T. A. Miller, A. NilsSon, R. W. Schoenlein, and A. M. LindenberG, Phys. Chem. Chem. Phys. 11, 3789 (2009).

25. R. W. Schoenlein, S. Chattopadhyay, H. H. W. Chong, T. E. Glover, P. A. Heimann, C. V. Shank, A. A. Zholents, and M. S. ZOLOTOREV, Science 287, 2237 (2000).

26. U. Bergmann, D. Nordlund, P. Wernet, M. Odelius, L. G. M. PetTERSSON, and A. NiLSSON, Phys. Rev. B 76, 024202 (2007).

27. T. Steinel, J. Asbury, J. Zheng, and M. Fayer, J. Phys. Chem. A 108, 10957 (2004).

28. S. Ashihara, N. Huse, A. Espagne, E. T. J. Nibbering, and T. ElsAesser, J. Phys. Chem. A 111, 743 (2007).

29. Z. Wang, Y. Pang, and D. D. Dlott, J. Phys. Chem. A 111, 3196 (2007).

30. J. LindNer, P. VÖHRINGER, M. S. PSHENICHNIKOV, D. CRINGUS, D. A. Wiersma, and M. Mostovoy, Chem. Phys. Lett. 421, 329 (2006).
31. S. Ashihara, N. Huse, A. Espagne, E. T. J. Nibbering, and T. ElsAesSer, Chem. Phys. Lett. 424, 66 (2006).

32. R. Rey, K. B. Møller, and J. T. Hynes, J. Phys. Chem. A 106, 11993 (2002).

33. A. LUZAR and D. CHANDLER, Nature 379, 55 (1996).

34. R. Rey, F. Ingrosso, T. Elsaesser, and J. T. Hynes, J. Phys. Chem. A 113, 8949 (2009).

35. C. HUANG, K. T. WikfeldT, T. TOKUSHIMA, D. NordLund, Y. HARADA, U. BERGMANN, M. NIEBUHR, T. M. WEISS, Y. HORIKAWA, M. LEETMAA, M. P. LJUNGBERG, O. TAKAHASHI, A. LenZ, L. OJAMÄE, A. P. LyUbartSEV, S. Shin, L. G. M. PetTERSSON, and A. NilsSON, Proc. Natl. Acad. Sci. 106, 15214 (2009)

36. W. C. RÖNTGEN, Ann. Phys. 281, 91 (1892).

37. G. E. Walrafen, M. S. Hokmabadi, and W.-H. Yang, J. Phys. Chem. 92, 2433 (1988).

38. H. IgLEV, M. SCHMEISSER, K. SimEONIDIS, A. THALLER, and A. LAUBEREAU, Nature 439, 183 (2006).

39. A. LindenberG, Y. ACREMANn, D. Lowney, P. HeimanN, T. Allison, T. Matthews, and R. Falcone, J. Chem. Phys. 122, 204507 (2005). 